auch die (nunmehr erwiesenermaßen nur noch vermeintlichen) Freunde wieder zurück.

Um das Bild des Romans bei Bernig aufzunehmen: Ein solch großer See, trotz der wunderschönen Bergbaunachfolgelandschaften in Form von Seen, worin schon einige sächsische Orte begraben liegen, fehlt für Sachsen, worin es sich selbst versenken könnte oder nach Meinung anderer versenkt werden sollte. Denn auch auf Sachsen lastet ja das Stigma, dass signifikante Kreise der Bevölkerung rechtsgerichtete Haltungen und ausländerfeindliche Meinungen vertreten. Verliehen u. a. von Wolfgang Thierse mit seinen „sächsischen Verhältnissen“, also Konsensabweichungen, oder von Stefan Schirmer in der Zeit vom 21. August 2015, wo er Sachsen als das „unsympathischste deutsche Bundesland" ausmachte und den Sachsen einen Säxit nahelegte, immerhin ja keine Leserzuschrift in einer bundesweiten sich als liberal sehenden Wochenzeitung, sondern eine Autorenmeinung. Und wie beim Bernig's schen Lehrer wird aus der Meinungsäußerung ganz unauffällig über die Zeit hinweg eine Tatsache, für die es sich nun in Sachsen zu verteidigen gilt. Wie kann das eigentlich heute geschehen, was ist da geschehen und ist Sachsen wirklich: Anders? Und was ist denn anders und warum?

Der Autor möchte einige Blickmöglichkeiten anbieten, die auf Widerlegung stoßen dürfen, aber vielleicht die eigene Sichtweise und die eigenen Diskussionszugänge zumindest anregen könnten.

Damit sind wir schon bei einem ersten wichtigen Punkt, der noch ganz unabhängig von Sachsen gilt: Diskussion. Was heißt das eigentlich? Schauen wir in Nachschlagewerke, so finden wir für Diskussion die Erläuterung als ein lebhaftes, oft kontrovers geführtes Gespräch über ein Thema oder Problem. Diskussion stammt vom lateinischen discussio und meint mithin ursächlich Untersuchung, Prüfung, Erörterung oder auch eine Sache besprechend erwägen. Bei den gesellschaftlichen Diskussionen besteht heutzutage manchmal noch Einigkeit um das Thema, auch wenn bereits hier oft auf die Empfehlungen von Arthur Schopenhauer zurückgegriffen wird und zwar auf seinen Kunstgriff 1: „Die Erweiterung. Die Behauptung des Gegners über ihre natürliche Grenze hinausführen, sie möglichst allgemein deuten, in möglichst weitem Sinne nehmen und sie übertreiben; seine eigne dagegen in möglichst eingeschränktem Sinne, in möglichst enge Grenzen zusammenziehen: weil je allgemeiner eine Behauptung wird, desto mehreren Angriffen sie bloß steht. Das Gegenmittel ist die genaue Aufstellung des puncti oder status controversiae." Jedoch wie man bereits in Talkshows im Fernsehen verfolgen kann, mangelt es uns oftmals an jeglichem Diskussionsstil. Heute wird nicht mehr diskutiert. Eine besprechende Erwägung setzt ja voraus, dass man verschiedene Argumente vorträgt. Dazu darf man dann nicht ins Wort fallen, denn der wirkliche Diskussionsteilnehmer ist ja auf die Vollständigkeit der Argumente aus. Man will in der Diskussion erwägen und mitnichten sofort verwerfen: ,ja aber...“. Insbesondere ergeben Diskussionen nur dann Sinn, wenn die Teilnehmer bereit sind, Argumente anderer in ihre eigene Problemlösungsfindung zu übernehmen. Diskussionsteilnehmer suchen in der Diskussion gemeinsam eine Lösung des Themas. Heutige Diskussionsteilnehmer sind aber Überwältiger und Selbstdarsteller zugleich, sie wollen den anderen mit ihren, immer wieder in Varianten vorgetragenen Argumenten, erschlagen und umstimmen, sie nehmen andere als die selbst schon vorab für richtig gehaltenen Argumente aber gar nicht erst gedanklich auf. Es gibt kein Richtig und Falsch zugleich mehr, nur weiß oder schwarz, und letzteres ist immer der andere.

Ein gutes Beispiel ist dafür das Wort „alternativlos“, weil es jede Diskussion vorab erwürgt. Wer es dennoch wagt, Alternativen wenigstens diskutieren $\mathrm{zu}$ wollen, steht plötzlich als dummer Mensch da, weil er ja gar nicht verstanden hat, dass es nichts $\mathrm{zu}$ diskutieren gebe. „Alternativlos“ ist das Aufgeben jeder demokratischen Position. Und wer vielleicht sich sportlich betätigt, der weiß, dass „alternativlos“ nie zum Sieg führt. Wer sich einmal verletzt hat beim Bergwandern, der bleibt nicht alternativlos liegen, sondern sucht sich zu retten. Es ist kein Geld da, wurde der Bevölkerung jahrelang vermittelt, Hartz IV wurde erfunden (wer Hartz IV bezieht, der weiß, dass kein Geld da ist, um ins Kino oder Theater zu gehen, eine Tageszeitung zu halten, sich also am kulturellen Leben, im Sinne der gesellschaftlichen Identität, zu beteiligen) - aber um den Austritt Griechenlands aus der EU zu verhindern, war plötzlich genug Geld da, der Bürger merkte es an immer neuen Scheinen aus dem Automaten. Die „Katastrophe“ für die EU, der Austritt Griechenlands, konnte bis jetzt abgewendet werden; hingegen „Großbritannien verlässt die EU, weil eine Mehrheit das politi- 
2 Dirk Schümer, in: Die Welt, 25. Juni 2016.

3 chrismon, Heft 1/2017.

4 Vgl. Trawny im Philosophie Magazin Februar/März 2017: „Das Grundgesetz hat keine narrative Aura."

Es gibt jedenfalls etwas, das ein Ensemble, ein Miteinander darstellt, das man vielleicht unmöglich beschreiben oder definieren kann, aber für das man sehr wohl das Wort "Volk“ benutzen kann. Es gibt eine Form der Gemeinschaft, die jeglicher individuellen Existenz vorausgeht und die die individuelle Existenz erst möglich macht. Jean-Luc Nancy

Etwas ist nur dann wichtig, wenn und weil wir Gründe haben, es für wichtig zu halten. Derek Parfit sche Versagen der Gemeinschaft nicht länger hinnehmen will. Dazu hat die Bundeskanzlerin mit ihren Alleingängen in der Flüchtlingspolitik maßgeblich beigetragen." ${ }^{2}$ Das eine gerettet, das andere verloren. Auch die Katastrophe, sofern überhaupt der Austritt eines Landes aus der EU eine solche ist, wird verarbeitet. Alternativen gibt es immer ${ }^{3}$ - das weiß eigentlich auch die Bundeskanzlerin, denn viele Sachsen hatten sich 1989 in Plauen, Leipzig und Dresden einfach getraut, vor (anders als heute geschulte) Polizisten zu treten und der Alternative zur real existierenden DDR den Weg zu öffnen. Das Suchen einer Alternative durch Menschen, die nicht Rücksicht auf ihr persönliches Wohlergehen nahmen (eindrucksvoll im SolidarnośćZentrum in Danzig erlebbar, hier spürt man förmlich den Mut, die Angst, die Freude, das Hintenanstellen persönlicher Belange der Menschen), hat letztendlich Angela Merkel zur - in den Augen der SPD nicht alternativlosen - Kanzlerin gemacht.

\section{Ist Sachsen anders?}

Die Antwort kann aus logischen Gründen nur lauten: Unbedingt. Der Begriff des Individuums setzt notwendig ein solches voraus. Dieses muss geradezu verschieden von anderen Individuen sein. Wären Stämme, die wir gern mal entdecken, Volksgruppen wie in Sachsen die Sorben oder Völker nicht von anderen verschieden, wären sie kein eigenes. Wir zeigen aber mit solchen Fragestellungen kein gutes Verhältnis zu unserer eigenen Zivilisation, wir haben kein Vertrauen zu uns selbst. Ulrich Baron zeigte in der „Welt“ vom 2. Juni 2008 am Beispiel der neu entdeckten 87 Mitglieder der Metyktire uns unser Missverständnis von Zivilisation auf, indem er auf Wissenschaftler hinwies, die vor Folgen warnten, wenn dieser Stamm mit uns in Berührung käme, weil das Immunsystem der Indios nicht auf Infektionen mit ihnen unbekannten Krankheiten eingestellt sein könnte - und er mit dem schönen Hinweis schloss: Was also sagt es über unser Zivilisationsverständnis aus, wenn man jedem Dschungelbewohner an der Pforte am liebsten rät, sobald und so weit in den Wald zurückzukehren wie nur möglich?

Wenn der Badener kein Württemberger sein will, so darf auch der Sachse eben Sachse sein. Beide, Badener und Sachsen, dazu Württemberger, Bayern, Sorben, Dänen in Schleswig-Holstein, Menschen polnischer Abstammung im Ruhrgebiet, Berliner türki- scher Abstammung usw. sind aber zugleich auch Deutsche. Und die Deutschen, soweit sie auch voneinander verschieden sind, sind offensichtlich gewillt, das Grundgesetz als gemeinsame Grundlage des Zusammenlebens $\mathrm{zu}$ akzeptieren (vielleicht weniger im Sinne eines Verfassungspatriotismus, Erfindungen sind immer schwer zu vermitteln und Patriotismus auf 146 Artikel mit vielen Absätzen ist eben schwerer als bei ursprünglich sieben, heute 34 Artikeln der Verfassung der Vereinigten Staaten ${ }^{4}$ ), weil es sich für alle lohnt, in Deutschland zu leben - selbst wenn sie ihre Nachbarn nicht leiden mögen, weil diese dick sind, die falsche Musik hören, eine andere Partei wählen, nicht vegetarisch essen, eine andere Hautfarbe haben, eine Burka als Zeichen der Abgrenzung tragen ... oder weil sie eben Sachsen sind.

Die, natürlich provokant anregend gemeinte und auch so beantwortete, Frage: „Ist Sachsen anders", scheitert bereits hier. Doch die Unklarheiten häufen sich, weil sie eben aus Pauschalisierungen erwachsen:

\section{Was ist Sachsen und wer ist Sachse?}

Das heutige Sachsen, ein Gebiet an der oberen Mittelelbe, der südlichen Lausitz und im Erzgebirge, hat mit dem Stammesherzogtum Sachsen, also dem Siedlungsraum des historischen Sachsenvolkes in Norddeutschland, nichts zu tun. Sachsen war lange Zeit von slawischen Stämmen besiedelt. Mit der Ostsiedlung durch vor allem Franken und Thüringer (Markgrafschaft Meißen 929) begann die Übernahme und Erweiterung slawischer Strukturen, übrigens wohl weitestgehend ohne große Konflikte oder gar Vertreibungen. 1089 kam die Markgrafschaft Meißen in den Besitz der Wettiner und mit dem Zugewinn infolge Aussterbens im Mannesstamm der sächsisch-wittenbergischen Linie des askanischen Herzogtums Sachsen-Wittenberg, welches seit der Goldenen Bulle 1356 die Kurwürde besaß und den Titel Herzog von Sachsen nach dem Sturz von Heinrich des Löwen erhalten hatte, erlangte 1423 die sächsische Kurwürde die meißnische Linie der Wettiner. Der Name Sachsen wurde dadurch allmählich auf die Markgrafschaft Meißen übertragen. Mit der Leipziger Teilung 1485 erfolgte dann die Trennung in eine ernestinische und eine albertinische Linie. Nach der Schlacht von Mühlberg 1547 gelangte die sächsische Kurwürde von den Ernestinern auf die Albertiner. 1806 wurde Sachsen Königreich durch seine Hinwendung zu Napole- 
on, 1815 verlor es im Wiener Kongress zwei Drittel seines Gebiets an Preußen (Österreich und Frankreich sicherten überhaupt den Fortbestand Sachsens; 1866 im Deutschen Krieg stand Sachsen auch auf der Seite Österreichs). 1918 wurde Sachsen Freistaat, in der Weimarer Reichsverfassung als Synonym für Republik begriffen, wobei zu beachten ist, dass im 17. und 18. Jahrhundert Republik auch für beispielsweise Monarchien usw. verwendet wurde; Freistaat hebt also bewusst ab von der Bindung an eine Dynastie; für das damals „Rote Sachsen“ (vergleiche die Verhängung des Belagerungszustandes über Sachsen durch die Reichsregierung) auch folgerichtig.

Sachsen ist nun nicht zu groß, aber doch innerlich verschieden. Mit der Zuschreibung, Sachsen sei anders und besonders unsympathisch, werden demnach nicht alle in Sachsen lebenden Menschen einverstanden sein. Zuallererst die Leipziger nicht, denn wie uns Oberbürgermeister Burkhard Jung, stammend aus Siegen und aus sächsischen Geldern sicherlich mehr als nur auskömmlich alimentiert, über den „Spiegel“ vom 20. Februar 2016 wissen ließ, sei seine Stadt aufgrund des Zuzugs aus der alten Bundesrepublik „vom Lebensgefühl her westdeutsch, zumindest sind wir weltoffen, gerade wegen unserer Messen, und nicht zu sächsisch“. Auch die Vogtländer sind nicht unbedingt Sachsen in ihrem Selbstverständnis, viele Görlitzer sehen sich als Schlesier, die Sorben sind auch nicht die mit der Fragestellung gemeinten Sachsen, die zugewanderten Bayern, Niedersachsen, Badener usw. dürften das Etikett Sachse in diesem Sinne strikt von sich weisen und die Bürger türkischer, vietnamesischer, griechischer, italienischer usw. Herkunft sowieso. Der Sachse, der gemeint ist, lebt irgendwo zwischen Dresden und Bautzen, zwischen Königstein und Freital - und er muss schon immer von hier sein.

Schaut man sich die Zuwandererzahlen an, so wird noch deutlicher, wer alles die Zuschreibung „Sachse“ im von Stefan Schirmer gemeinten Sinne sofort ablehnen dürfte. Gemeinhin wird ja nach den Wanderungssalden geschaut, die für Sachsen, aber auch für die anderen ostdeutschen Bundesländer eher negativ ausschauen, es gibt also einen markanten Bevölkerungsverlust (für Sachsen zwischen 1991 und 2013 von über 630.000 Menschen $^{5}$ ). Dieser Wanderungssaldo reicht aber allein nicht für eine Aussage über den Wandel der Bevölkerungsstruktur aus, bei beispielsweise einem Saldo von minus
100.000 können 1.000 .000 weg- und 900.000 zugezogen sein oder auch nur 100.000 wegund 0 zugezogen sein. Daneben sind noch die Bevölkerungszusammensetzung, das Alter usw. von wesentlicher Bedeutung.

Sucht und rechnet man also nur die Zuzüge, so ergibt sich ${ }^{6}$ von 2002 bis 2015 ein Zuzug nach Sachsen von insgesamt 1.020.456 Menschen, davon 366.671 Nichtdeutsche, wobei ein starker Anstieg 2014 und 2015 (ungefähr Verdopplung gegenüber den vorhergehenden Jahren) zu verzeichnen ist. Demgegenüber standen 1.006.437 Wegzüge aus Sachsen, davon 273.533 Nichtdeutsche; wobei die Wegzüge insgesamt leicht sinken. Das heißt, allein die letzten 14 Jahre gab es einen Bevölkerungsaustausch von rund einem Viertel der Gesamtbevölkerung (dies gilt auch dann, wenn man die Rückkehrer nach Sachsen bedenkt, denn auch diese haben neue Prägungen erfahren). ${ }^{7}$ Ganz sicher darf man davon ausgehen, dass all diese Menschen hierher gezogen sind, weil Sachsen lebenswert ist und nicht deshalb, weil sie bei PEGIDA mitdemonstrieren wollen. Die verunglimpfend gemeinte Zuschreibung „Sachse“ in der „Zeit" für alle hier in Sachsen Lebenden durch einen Journalisten, zu dessen Ethos eigentlich doch eine gründliche Recherche und gedankliche Überlegung, im Gegensatz zu einer eigenen Meinungsäußerung, gehören sollte, ist daher verfehlt und trägt wenig zu einer Versachlichung der Diskussion bei.

Der Ausländeranteil in Sachsen ist mit Bestimmtheit gering; laut Statistischem Landesamt betrug er 2010114.000 Menschen; nach dem Mikrozensus 2005 geschätzt 219.000 Menschen mit Migrationshintergrund (um die 5 Prozent an der Gesamtbevölkerung, Bundesdurchschnitt 19,6 Prozent $^{8}$ ). Daraus aber den Schluss ziehen zu wollen, dass die Sachsen nicht über den Zuzug von Migranten diskutieren dürfen (Sachsen lesen im Übrigen auch Zeitung, sehen fern oder benutzen das Internet, sie haben also durchaus ein Verständnis von dem, was um sie herum passiert) ist genauso falsch, wie nur Anwohner im Umkreis von Atomkraftwerken zur Diskussion über Atomenergie zuzulassen. Ein Pfarrer muss nicht erst tot sein, um eine Beerdigung richtig durchführen zu können.

Interessant erscheint in diesem Zusammenhang die Überlegung, wie wir eigentlich zukünftig mit unserer besonders bedrückenden Geschichte der Jahre 1933 bis 1945 umgehen wollen. Wie gerade gesehen, werden wir ja immer mehr Deutsche mit anderen Wurzeln.
525 Jahre Deutsche Einheit, Statistische Ämter des Bundes und der Länder; prozentual war der Bevölkerungsrückgang allerdings in Sachsen-Anhalt, Thüringen und Mecklenburg-Vorpommern am höchsten.

6 Eigene Berechnung nach Angaben des Statistischen Bundesamtes, Fachserie 1, Reihe 1.2, 2015.

7 Auch in den 1990er Jahren gilt dies, so zogen allein 1991 aus Sachsen über 72.000 Menschen weg, vgl. Binnenwanderung zwischen Ostund Westdeutschland seit 1990 - Darstellung der Zahl und Struktur, Determinanten, zukünftige Entwicklung; Yasmin Büttgen, Universität Trier. Wenn man dies hochrechnet, käme man auf einen Bevölkerungsaustausch von ca. 50 Prozent.

8 www.migrasax.de; dies gilt auch 2013 laut der Publikation „25 Jahre Deutsche Einheit, Statistische Ämter des Bundes und der Länder"; allerdings liegt Sachsen bei den ostdeutschen Flächenländern damit nur knapp hinter Brandenburg und deutlich vor den anderen dreien. 
9 Vgl. die Aufsätze in Frank Lothar Kroll/ Hendrik Thoß (Hrsg.): Zwei Staaten, eine Krone. Die polnisch-sächsische Union 1697-1763, Berlin 2016 .

Nichts passiert ohne zureichenden Grund. Gottfried Wilhelm Leibniz
Schon jetzt haben wir in Deutschland, je nach Statistik, rund 20 Prozent Einwohner mit Migrationshintergrund, dies sind bei 82 Millionen Bundesdeutschen also über 16 Millionen aller Deutschen. Wenn sich Deutschland seiner besonderen Verantwortung stellen muss, wie machen das dann diese 16 Millionen Deutschen? Welche Verantwortung können sie überhaupt tragen? Wenn wir uns als Zuwandererland bezeichnen wollen, wie wirkt sich das auf die Übernahme historischer Verantwortung aus?

\section{Was ist anders in Sachsen?}

Wie wäre eigentlich die Frage 1517 intendiert gewesen, als das Kurfürstentum Sachsen der Hort der Reformation war - und u. a. hierdurch einen wesentlichen Beitrag zur deutschen Kulturnation, die Jahrhunderte vor dem Nationalstaat existierte, leistete? Oder 1918/1919, als das „Rote Sachsen“ Furore machte? Oder am 17. Juni 1953, als in den Kreisen Görlitz und Niesky für wenige Stunden das SED-Regime beseitigt werden konnte? Oder 1989, als die Friedliche Revolution von Sachsen aus sich Bahn brach? Natürlich muss man bei dieser positiven Aufzählung auch das „Braune Sachsen“ in Rechnung stellen, denn in Sachsen erzielten die Nationalsozialisten relativ schnell hohe Zustimmungswerte. Aber es ist falsch und einseitig, Sachsen eine schon immer starke rechtsradikale Gesinnung zu unterstellen. Frank Richter, ehemals Direktor der Sächsischen Landeszentrale für politische Bildung, verweist in der „Welt“ vom 26. August 2015 für die „Anfälligkeit“ von Kreisen der Bevölkerung für Gedankengut rechtsextremer Parteien auf folgende interessante Überlegung: „Die DDR hat sich als antifaschistischer Staat definiert. Der Nationalsozialismus wurde als große Katastrophe der deutschen Geschichte dem Kapitalismus und Imperialismus zugeordnet. Und damit hatten die Deutschen in der DDR per definitionem nichts zu tun. Eine ehrliche und offene und persönliche Aufarbeitung der schuldhaften Verstrickungen in der Nazizeit hat es nicht gegeben, jedenfalls nicht in meiner Umgebung. Es wurde viel geschwiegen und wenig darüber gesprochen. Dieses kommunikative Gedächtnis wird von einer Generation an die nächste weitergegeben. Die Gesprächs- und Diskussionskultur, die eine offene Gesellschaft auszeichnet, ist schwach ausgeprägt. Das spielt den Rechtsextremen in die Hände. Die Abwehr des Fremden sichert vermeintlich die schwach ausge- prägte Identität, die ihrerseits mit mangelnder Geschichtsaufarbeitung zu tun hat."

Auch den Begriff „Rechts“ zu diskutieren ist wohl müßig: wer „Links“ sein will, braucht denknotwendig den sprachlichen Gegenspieler „Rechts“. Auch „konservativ“ (den Konservativen geht es im ersten Blick immer darum: was sollte bewahrt werden - es schließt aber Neuerungen keinesfalls aus, nur eben fußend auf schon Bewährtem) und „rechtskonservativ“ sind zulässig; „rechtsextrem“ und „linksextrem“ sollten jedoch zu denken geben.

Sachsen hat eine lange reichhaltige historische Tradition und war eines der maßgebenden Länder im Heiligen Römischen Reich Deutscher Nation. Hieraus folgt zwangsläufig Selbstbewusstsein. Mit der Kurwürde, die eben nur die sieben, später neun Kurfürsten zur Königswahl berechtigte, war es exponiert unter geschätzt um die 300 deutschen Kleinstaaten nach dem Westfälischen Frieden. Der sächsische Herrscher musste auch nicht ein schwacher deutscher König werden - dies lehnte der sächsische Kurfürst wohlwissend ab. Sachsen vermochte es hingegen, auf anderen Wegen außenpolitisch mitzuwirken zum Beispiel, indem der sächsische Kurfürst es schaffte, sich zum polnischen König krönen zu lassen. ${ }^{9}$ Sachsen ist eines der wenigen deutschen Bundesländer, die auf ein sehr langes Selbstverständnis als Staat (und zwar eben als Staat, der die Politik im Alten Reich und ansatzweise in Europa mitbestimmte) zurückgreifen können, denken würde man zuallererst noch an Bayern. Von daher muss Sachsen ein starkes Traditionsverständnis haben, welches u. a. beim Aufbau ab 1990, zuerst durch „König Kurt“, durchaus gezielt angesprochen wurde und weder den sächsischen Einwohnern noch dem Ansehen der Bundesrepublik schadete. Mit Sachsen zeigte sich, dass es eben doch geht. Historisch gesehen besitzt Sachsen auch eine starke Südbindung - zu Bayern, zu Österreich, welches ja den Fortbestand von Sachsen überhaupt sicherte. Insofern war Sachsen aber auch oftmals gegen ein immer übermächtiger werdendes Preußen, also gegen die Hauptstadtpolitik im Reich, positioniert.

Dieses Traditionsverständnis, welches natürlich immer nur ein Gesamthinweis sein kann und nicht am Einzelnen festzumachen ist, insofern bleibt auch vieles glücklicherweise dem Zufall überlassen, hat nicht nur tiefe Wurzeln, sondern erhält auch immer neue Anker. Traditionsverständnis ist ebenso nicht nur ein „rechtes“ Domizil, sondern in 
der Bevölkerung weit verbreitet, je nachdem, für welche Absichten man es einsetzen möchte. So wäre z. B. auch an den Einsatz grüner und anderer Apologeten zur Verhinderung der Dresdener Waldschlösschenbrücke zu denken, um das Dresdner Elbtal, welches es trotz Brücke weiterhin unbestritten gibt, zu schützen. Auch hier wurde auf Tradition verwiesen, gebracht hat es lediglich den Verlust des Welterbetitels und genutzt wird die Brücke intensiv.

Sicherlich wurde und wird ein „sächsisches Bewusstsein“ aber auch vom Staat gefördert. Sachsen war der sechste Bundesstaat und der erste ostdeutsche überhaupt ${ }^{10}$, der seit 1992 mit dem „Tag der Sachsen“ einen eigenen Heimattag hat ${ }^{11}$, die „Landesmutter“ Ingrid Biedenkopf veranlasste „ihre“ Sachsen zur Suche nach einer Sachsenhymne - und die Staatskanzlei druckte von den Einsendungen ein Buch. Seit 1997 gibt es die Sächsische Verfassungsmedaille, gegen die Kürzung von Denkmalpflegemitteln erwächst sofort starker Protest wie zuletzt bei Petition „Denkmale in Sachsen retten“ zum Regierungsentwurf des Sächsischen Haushaltsplanes 2017/2018. Bergmannsaufzüge, Mettenschichten und vieles mehr tragen zu einem reichen Kulturerbe ebenso bei wie zum Selbstverständnis der (Neu-)Sachsen. Das Landesamt für Denkmalpflege Sachsen hat für und im Freistaat eine ganz überragende Bedeutung. Auch heute wird der Sachsenstolz, der bisweilen absurde Züge trägt, etwa wenn sich ein Unternehmen „Sachsenfett“ nennt, erfolgreich angesprochen. So verwies die „Süddeutsche Zeitung“ am 17. Mai 2010 auf Folgendes: „Es reicht, wenn er [gemeint ist Ministerpräsident Stanislaw Tillich] sich an das Biedenkopf'sche Erfolgsrezept hält: einfach die stolze sächsische Seele streicheln. Auf Wahlplakaten steht neben seinem Konterfei in dicken Lettern: ,Der Sachse،.“

Tradition wird oftmals sichtbar an einem Punkt oder Ort, der sich im Gedächtnis einzuprägen vermag. Deutscher Karneval ist mit Köln, Mainz, Düsseldorf verbunden, und Hamburg ist eben das Tor zur Welt. Hamburg und Dresden als Partnerstädte vertreten in ihrem Stadtbild auch zwei ganz unterschiedliche Baukonzepte: Hamburg feierte kürzlich die Einweihung des 2016 fertiggestellten Konzerthauses Elbphilharmonie (nicht zu verwechseln mit der Elbland-Philharmonie Sachsen); Sachsen hingegen weihte am 30. Oktober 2005 die mit Spenden aus aller Welt wiedererrichtete Frauenkirche ein, verbunden mit einem Orgelstreit über das richtige Traditionsverständnis. Für Sachsen ist Dresden der Ort, der Gedächtnis prägt. Im durchaus reibungsvollen und manchmal ungewollten Nach- und Zusammenwirken haben die DDR, dann die Regierung des Freistaates und insbesondere auch die Dresdner Einwohner die Landeshauptstadt $\mathrm{zu}$ einer über die Grenzen Deutschlands hinweg bekannten und berühmten Residenzstadt wieder erstehen lassen; auch wenn die Dresdner in großer Selbstverliebtheit ihr bauliches Erbe auf einen imaginären Zeitpunkt hin ausrichten. Das hat mehrere Auswirkungen.

Die Kulisse: Der Transport von Meldungen braucht heutzutage Bilder, Medien brauchen Bilder. Die PEGIDA-Demonstrationen sind eben nur vor der Kulisse Dresdens wirkmächtig, sowohl für Pegida selbst als auch für das Fernsehen. Und mit Kulisse ist ein Ort gemeint, der eine Bewegungsmöglichkeit bietet. Semperoper, Theaterplatz, Frauenkirche, Kreuzkirche, Georgentor, Fürstenzug, Brühlsche Terrasse - das gibt es so sonst nirgendwo in Sachsen. Wo könnte man sowohl für die eigene Anhängerschaft als auch für die Medien, was das Kommen ja eher stärkt als schwächt, über Jahre hinweg attraktiver wahrgenommen werden? Würde das funktionieren in Leipzig vor dem Alten Rathaus oder wenn man um den Augustusplatz liefe? Oder in Bautzen wohl nur am Kornmarkt, was ja auch so ist; in Chemnitz ist vermutlich kein Ort dauerhaft attraktiv genug. PEGIDA ist auch ein Produkt der Bilder, die nur in Dresden tatsächlich funktionieren.

Residenzstadt und Publikum: Dresden war über Jahrhunderte hinweg eine europäische Residenzstadt mit einer glanzvollen Kulturund Prachtentfaltung, mit einem ihren Einwohnern $\mathrm{zu}$ eigen gemachten Kulturgefühl. „Das Alte Dresden“ von Löffler hatte man. Theater- und Opernanrechte wurden vererbt. Bildung war kein zwanghaftes Muss, sondern entsprach innerer Freude. Man findet das alles im „Turm“ von Uwe Tellkamp. Als vergleichbare deutsche Residenzstadt würde man vielleicht noch Weimar ansehen können; und eben nicht Potsdam, welches ja Jagdresidenz ab ca. 1660 und dann vor allem auch Garnisonsstadt war - Sachsen verkaufte seine Soldaten gegen Vasen. Zudem ist Potsdam wesentlich jünger, was man auch bei Mannheim oder Karlsruhe schon in ihrem Straßennetz ablesen kann. Residenzstädte führen jedoch ein Eigenleben. Sie haben ein langes Traditionsverständnis, sie definieren sich durch ihr Kulturleben (welches eben dem Kulturbedürfnis der Einwohner ent-
10 Tag der Franken seit 2006; Brandenburg-Tag seit 1995 , seit 2004 nur noch zweijährlich; Mecklenburg-Vorpommern-Tag seit 2000, seit 2006 nur noch zweijährlich; Tag der Niedersachsen seit 1981, seit 2015 nur noch zweijährlich; NordrheinWestfalen-Tag seit 2006, seit 2012 nur noch zweijährlich; Rheinland-Pfalz-Tag seit 1984, Saarlandtag siebenmal zwischen 1988 und 2007; Sachsen-Anhalt-Tag seit 1996; Schleswig-HolsteinTag von 1992 bis 2012 zweijährlich; Thüringentag seit 1996, seit 2007 zweijährlich; Hessentage seit 1961, Heimattage Baden-Württemberg seit 1978 .

111914 gab es schon den sogenannten Sachsentag. Weiter sei auf die Bundeslandsmannschaft Sachsen verwiesen, vgl. Hans Haferland: Für Einheit in Freiheit Die Bundeslandsmannschaft Sachsen 1954-1993, Dresden 2005 (Herausgeber war der Sächsische Landtag, der Verfasser Dr. Hans Haferland wurde 2010 mit dem Verdienstorden des Freistaates Sachsen ausgezeichnet), sowie auf die von Mitgliedern der ehemaligen Bundeslandsmannschaft Sachsen gegründete Stiftung Land Sachsen, errichtet $1975 \mathrm{mit}$ Sitz in Coburg, mittlerweile aufgelöst mit Übertragung ihrer Sammlung an die Gemeinde Großhartmannsdorf. 
Eine Vorstellung von Gerechtigkeit, die der Freiheit - insbeson-

dere der Redefreiheit - keine Bedeutung gibt, ist wahrscheinlich nicht vertretbar. Amartya Sen

12 Hardeep Singh Puri, Die Zeit, 5. Januar 2017.

13 Die „Oberhessische Presse“ vom 22. November 2016 vermeldete: „Die Sachsen haben wenig Vertrauen in die Demokratie und weisen eine starke gruppenbezogene Menschenfeindlichkeit auf - vor allem gegenüber Muslimen. Das sind Ergebnisse des sogenannten ,SachsenMonitors', der am Dienstag in Dresden vorgestellt wurde. Im Auftrag der Staatsregierung hatte das Meinungsforschungsinstitut dimap von Anfang August bis Anfang September erstmals politische Einstellungen der Bevölkerung und ihre Haltung zur Demokratie abgefragt. [...] Die Ergebnisse zu extrem rechtem, gruppenbezogen menschenfeindlichem und rassistischem Gedankengut nannte Richter ,alarmierend'. 69 Prozent der Befragten glaubten nicht, dass die in Deutschland lebenden Muslime, unsere Werte' akzeptierten. 39 Prozent sind dafür, Muslimen die $\mathrm{Zu}-$ wanderung $\mathrm{zu}$ untersagen. 18 Prozent der Sachsen glauben, dass Deutsche , anderen Völkern von Natur aus überlegen" sind." Auch hier wird zusammengerührt, was nicht immer zusammenpasst: Die 69 Prozent, die sicherlich eine Ansicht vertreten, die auch spätestens seit der Silvesternacht 2015/2016 in Köln von breiteren Kreisen geteilt werden wird, zu den extrem rechtem, gruppenbezogen menschenfeindlichem und rassistischem $\mathrm{Ge}$ dankengut zuzurechnen, ist schon in der Fragestellung und "wissenschaftlichen“ Schlussfolgerung unlauter. Wenn ich feststelle, dass andere Menschen andere Werte akzeptieren, sagt das noch nicht einmal aus, wie ich zu diesen Menschen ste- spricht), sie sind Verwaltungssitz und damit ist ihr Bürgertum auch von einer Beamtenschaft mit getragen, welche von ihrem Selbstverständnis her schon die bestehende Ordnung nicht permanent anzweifelt. Dieses Beharrungsvermögen widerspricht keineswegs der steten Freude an Neuem: Jeder Dresdner weiß, dass ohne italienische Architekten, Maler, Sänger usw. Dresden als solches gar nicht denkbar wäre. Aber es geht eben etwas leiser zur Sache, es wird nicht der Wechsel, sondern das Einpassen mit eigenem Akzent gesucht. Einem zumal noch von Berlin kommenden Umschwung, den man selbst nicht mit gestalten kann und der sichtbar Probleme mit sich bringt, steht man zwangsläufig skeptisch gegenüber. Übertragen könnte man an den Paragraph 34 des Baugesetzbuches denken, wonach Vorhaben zulässig sind, wenn sie sich in das Vorhandene einfügen.

Montagsdemonstration: Eine gewisse Autorität leihen sich Protestbewegungen dann, wenn sie die „Wendetradition“ der Montagsdemonstrationen, die es zuerst in Leipzig gab, aufnehmen; eine Strategie, die übrigens regelmäßig bis in die heutige Zeit angewandt wird: „Montagsdemonstrationen“ fanden so gegen das Kommunalabgabengesetz, gegen Schulschließungen und gegen den Sparkassenverband statt. Gerade die Okkupation des Montags durch PEGIDA ist natürlich ein Umstand, der besonders bei den Leipzigern breiten Unmut hervorrufen musste.

Idee der Friedlichen Revolution: „Wende“ ist eigentlicher kein guter und mindestens viel zu kraftloser Begriff. Eingeführt wurde er zudem durch Egon Krenz am 18. Oktober 1989 in seiner Antrittsrede. Er machte damit deutlich, was er wollte, aber eben die Menschen auf den Straßen gerade nicht: Eine nötige Wende durch die SED. Mit Friedlicher Revolution scheint hingegen der bürgerschaftliche Ansatz, der aufgebrachte Mut, der gerade friedliche und nicht gewaltsame Weg und das erreichte Ziel, nämlich die Zerschlagung des vorherrschenden Gesellschaftsmodells, richtig betont. Wobei das Endziel offen und diffus war, man wusste eher was man nicht mehr wollte, aber weniger, was kommen sollte. Ein wie auch immer zu begehender „Dritter Weg“ schwebte manchem vor. Die ersten Ziele waren auch den Transparenten zu entnehmen: „Für ein offenes Land mit freien Menschen“, „Freiheit“, „Wir wollen raus“, „Wir brauchen Reformen“, „Für Reformen und Reisefreiheit gegen Massenflucht vor allem Frieden“ oder „Reisefreiheit - Meinungsfreiheit - Pressefreiheit“ und „Wir sind ein Volk“, um nur einige zu nennen. Ausgangspunkte für die DDR waren Leipzig, Plauen und Dresden. Zweierlei wird damit deutlich, was auch heute noch nachwirkt bei manchem im Osten Deutschlands, aber über die sächsischen Grenzen hinaus:

a) Wichtige Forderungen waren Freiheit, Meinungsfreiheit, Pressefreiheit, und man war stolz auf seinen Mut, auf die Straße gegangen zu sein und dem System getrotzt, es letztendlich zersprengt $\mathrm{zu}$ haben. So etwas prägt sich ein, besonders, da man die Polizei und Kampfgruppen nicht als Einheiten zur Absicherung der Demonstrationen sah, sondern sie Gegner waren; vereinzelt, so in Plauen und Dresden, kam es ja auch zu gewalttätigen Übergriffen der Staatsmacht. Was man aber damals sich getraute (Demonstrationsrecht) und erkämpfte (Meinungsfreiheit, Pressefreiheit), und welches sich zudem noch mit dem Grundgesetz deckt, das will man auch heute, auch wenn die Meinung wiederum nicht „den Regierenden“ passt, nicht aufgeben. Die Pressefreiheit, unter der eine unabhängige und nicht meinungsgeleitete Berichterstattung verstanden wird, hat sich in den Köpfen der Menschen als hohes Gut etabliert, auch wenn sie eben nicht so erfahren wird - auch im Ausland. „Eure politischen Führungen fallen einem emotionalisierten Journalismus zum Opfer."12 Ein Rechtfertigungsdruck schlägt dann eher ins Gegenteil um. Am 29. Dezember 2015 vermeldete „Tag24“ dazu: „Sachsens früherer Ministerpräsident Kurt Biedenkopf (85, CDU) hat indirekt Verständnis für das islamund fremdenfeindliche PEGIDA-Bündnis geäußert. ,Die PEGIDA-Demonstrationen sind Ausübung eines ganz entscheidenden demokratischen Grundrechts, nämlich demonstrieren zu dürfen. Und es gibt genug Gründe in Ostdeutschland, nicht nur in Sachsen, sondern in Ostdeutschland, warum die Bevölkerung über diesen starken Flüchtlingszustrom beunruhigt ist', sagte Biedenkopf am Dienstag in einem Interview von Deutschlandradio Kultur. [...] Der wichtigste Grund sei, dass sie ,keinerlei Erfahrung، damit habe. Die Menschen im Osten hätten zudem 25 Jahre eine völlige Umstellung ihres Lebens bewältigt, ,und zwar in einer Tiefe, wie es sich Westdeutsche überhaupt nicht vorstellen können'."13

b) Aus dem 1989/1990 anfangs offenem Ziel, was eigentlich kommen soll, wurde der Ruf unter dem Slogan „Wir sind ein Volk“ nach der Wiedervereinigung laut. In den Augen des Ostens meinte wohl bei manchem aber 
Wiedervereinigung zweier deutscher Teile die Wiedervereinigung zu Deutschland und eben nicht eine Wiedervereinigung zur schon vorhandenen Bundesrepublik, denn „Für viele Ostdeutsche ist die Wiedervereinigung noch immer nicht abgeschlossen."14 Dieses unterschiedliche Bild und Erwartungshaltung zum Land, in dem wir leben, reflektieren ebenso diejenigen, die „anders“ sind - und gerade darum politisch einbezogen und nicht ausgegrenzt werden dürfen (was ja zur sog. Denkzettelwahl führt), denn: „Unsere Gesellschaften teilen sich heute nicht mehr in Ausbeuter und Ausgebeutete, sondern das entscheidende Kriterium ist, ob man sich ausgeschlossen fühlt. Und Ausgeschlossenheit wird von der extremen Rechten politisiert." ${ }^{15}$

Konstatieren könnte man hieraus, dass offensichtlich „die Sachsen“ für eine einmal gewonnene Ansicht, dass die Welt da draußen sich nicht mit dem deckt, was von ihr berichtet wird, sei diese Ansicht nun richtig oder falsch bzw. gesellschaftlich akzeptiert oder diskreditiert, tendenziell eher bereit sind auf die Straße zu gehen. Die Menschen akzeptierten keine Genehmigungsdemokratie mehr $^{16}$ und etwas verändern $\mathrm{zu}$ wollen ist nicht generell schlecht, nur die Art und Weise, erinnert sei an den 3. Oktober 2016, wäre zu hinterfragen. ${ }^{17}$ Das darf aber nicht dazu führen, dass hierhinein alle „rechten“ Probleme verschoben und auf Sachsen klammheimlich übertragen werden. Daher sei daran erinnert, dass die AfD zwar auch in Sachsen vergleichsweise starken Zuspruch genießt, jedoch ihre Gründung in Berlin erfuhr und zwar durch den West-Berliner Bernd Lucke, Professor für Makroökonomie in Hamburg, was auch auf den Impuls für die Parteigründung hinweist, nämlich die Kritik an der Euro-Rettungspolitik. ${ }^{18}$ Die erste öffentliche AfD-Versammlung fand in Oberursel im Taunus statt, weitere Führungspersönlichkeiten waren bzw. sind Konrad Adam aus Wuppertal, Jörg Meuthen aus Essen und Frauke Petry aus Sachsen. Doch auch hier lohnt es genauer hinzuschauen, bevor man verallgemeinert: Frauke Petry wurde zwar 1975 in Dresden geboren, lebte dann aber bis 1989 in Schwarzheide in der Niederlausitz und zog noch vor dem Fall der Mauer nach Bergkamen bei Dortmund. 1995 legte sie dort das Abitur ab und war bis über 2004 hinaus an der Universität Göttingen, wo sie auch promovierte. Sozialisiert hat sie sich also mitnichten in Sachsen. Sie ist also eine der oben genannten Rückkehrer, die sich auch in Sachsen wirt- schaftlich betätigten; auch zur Selbstständigkeit gehören Mut und Können und vom Mittelstand profitiert unser Land.

Allein dieses Beispiel reicht eigentlich aus um zu zeigen, dass es völlig unzulässig ist, von einzelnen Menschen, seien sie nun tüchtig oder nicht, auf eine Volksgruppe oder dergleichen $\mathrm{zu}$ schließen. Es ist reinweg auch Zufall, wer wo mit wem wie lange lebt. Kein ernstzunehmender Mensch käme auf die Idee, alle Bürger Ludwigshafens seien als Kanzler berufen. Auch alle drei NSU-Mitglieder, die sich in Zwickau und eben nicht in Eberswalde versteckten, stammen aus Thüringen (Eisenach und Jena) und waren bis nach 1997 dort aktiv. Sachsen war insofern Versteck und nicht Nährboden. Dieser kleine Exkurs soll aber nur zeigen, dass die Journalisten nicht gut daran tun, Bundesländer zu stereotypisieren.

Was sagen die Zahlen zum „rechten Sachsen“? Bei den Landtagswahlen 2014 erreichte die NPD in Sachsen 4,95 Prozent, in Thüringen 2014 3,6 Prozent und in Mecklenburg-Vorpommern 20163 Prozent. Überall sonst lag sie deutlich unter 3 Prozent. Nirgends ist sie aber im Landtag vertreten. Insofern ließe sich schlussfolgern, dass das Wählerbewusstsein auch in Sachsen gegenüber Parteien, die mit verfassungsfeindlichen Zielen in Verbindung gebracht werden können, gewachsen ist. Nicht zuletzt gibt der Autor hier ebenso zu bedenken, dass sich die NPD gerade auf Sachsen konzentrierte - so zog der rechtsextreme Verlag Deutsche Stimme aus Bayern nach Riesa. Die AfD erzielte ihre besten Landtagsergebnisse in Sachsen-Anhalt 2016 mit 24,2 Prozent; in Mecklenburg-Vorpommern 2016 mit 20,8 Prozent; in Baden-Württemberg 2016 mit 15,1 Prozent; dann folgen Berlin 2016 mit 14,2 Prozent; Rheinland-Pfalz 2016 mit 12,6 Prozent; Brandenburg 2014 mit 12,2 Prozent; Thüringen 2014 mit 10,6 Prozent; dann erst kommt Sachsen 2014 mit 9,7 Prozent, und nach Sachsen rangieren nur noch Hessen, Hamburg und Bremen (wobei natürlich die unterschiedlichen Jahreszahlen zu beachten sind, sicherlich wären die Wahlergebnisse 2016 für die AfD in Brandenburg, Thüringen und Sachsen besser ausgefallen). In Sachsen ist die CDU eine feste Bank - bisher. Seit 2004 regiert sie aber in Koalition mit der SPD oder der FDP, so dass auch die Aussage, die CDU würde alleine bestimmen, schlicht falsch ist. Was SPD oder FDP aus den ihnen gegebenen Möglichkeiten natürlich machen, ob wie bei der FDP Öffnungszeiten von Videotheken und Autowaschanlagen wirklich
Ich glaube, dass eine rein ökonomische Gemeinschaft ohne eine Gemeinschaft des Herzens im Sinne eines gemeinsamen Ziels zum Scheitern verurteilt ist. Martha Nussbaum

he. Immerhin findet die Studie auch einen Schluss über die Grenzen Sachsens hinaus: „Man soll sich auch keine Illusion machen, dass diese ganzen Einstellungen nur auf Sachsen begrenzt sind", sagte Schlinkert. Im Freistaat seien sie „vielleicht in manchen Ausprägungen etwas stärker als im Rest der Bundesrepublik“. Befragungen zeigten aber immer wieder, dass rund die Hälfte aller Deutschen der Aussage „Es macht mir Angst, dass so viele Flüchtlinge kommen“ zustimmten. An anderer Stelle verwies Biedenkopf darauf, dass die Demokratie solange nicht gefährdet sei, wie man an sie glaubt. Das macht aber auch nochmal die Verantwortung der Regierenden deutlich. Rechtsgrundsätze sind nur geronnene Erfahrung und damit lebendiges kulturelles Gedächtnis - sie sind ein gesellschaftlicher Kitt, der aber nicht einer Beliebigkeit unterworfen werden darf.

14 Stefan Locke, in: Frankfurter Allgemeine Zeitung, 10. November 2016.

15 Gilles Kepel, in: Die Zeit, 5. Januar 2017

16 Andreas Zielcke, in: Die Zeit, 10. November 2016.

17 Raphael Thelen, in: Die Zeit, 12. Mai 2016.

18 Dass Bernd Lucke mit seinen ökonomischen Überlegungen nicht allein stand, zeigt neben anderen Initiativen, die vielfältige Unterstützung durch Wirtschaftsprofessoren fanden, ein im September 2013 veröffentlichter Aufruf, in dem der EZB rechtswidrige monetäre Staatsfinanzierung vorgeworfen wurde. Diesen Aufruf unterzeichneten 136 deutsche Wirtschaftsprofessoren, darunter Roland Vaubel, Hans-Werner-Sinn und Georg Milbradt. 
19 Thomas Aussheuer, in: Die Zeit, 25. Mai 2016 über Trumps Wählerangebot.

Die Sprache ist die grundlegende soziale Institution. John Searle

Ergebnisse der Landtagswahlen in Sachsen (Angaben in Prozent) ein wichtiges Anliegen der Bürger sind, muss deren Führungspersonal entscheiden.

Die Ergebnisse der Landtagswahlen (siehe Tabelle) verdeutlichen nicht nur die bis jetzt noch tragende CDU-Dominanz in Sachsen, sondern verweisen gleichzeitig auf ein den Osten (neben den östlichen Bundesländern auch eingeschlossen andere Staaten wie Polen etc.) vom Westen unterscheidendes Merkmal: die wenn nicht fehlende, so doch wesentlich geringere Parteibindung.

Solange die Menschen eine Orientierung in den Parteien finden, mit denen sie sich identifizieren können, ist die Gefahr von großen Stimmungsumschwüngen gering. Insofern ist es sicherlich für eine Partei besser, ihr Profil auch für kritische Gruppen offen zu halten, als eine neue Partei durch eine eigene thematische Verengung entstehen zu lassen. Es ist für den Menschen leichter, auch mal so sein zu dürfen und bleiben zu können, wie er ist, er will auch mal hören: „Bleib so wie du bist. Du musst kein Opfer mehr bringen, ich respektiere dich auch so. ${ }^{19}$ Und es ist für den Menschen zudem leichter, einer Idee sein Gehör zu schenken, als immer wieder von Sparvorschlägen und Solidaritätsaufrufen gemartert zu werden. Diese sind per se nicht falsch, aber die Idee: wozu, wie schön haben wir es dann, was hat der Einzelne konkret davon - die geht oftmals unter. Und zumindest viele Menschen im Osten Deutschlands haben noch den Klang einer Idee aus den Jahren 1989/1990 im Ohr und würden sich einer neuen nicht verweigern. Dies ist Gefahr, aber auch Möglichkeit zugleich, man muss es nur sehen, ergreifen und das Feld besetzen wollen. Ein Negativbeispiel ist sicherlich Russland, welches mit seiner Krim- und Ukrainepolitik, überhaupt der Politik zurück zur alten Großmacht, die Lebensbedingun- gen der Menschen nicht verbessert, es aber versteht, sie allein durch die Idee eines wieder „großen starken Russlands“ von ihren Alltagssorgen abzulenken und für die Regierung einzubinden. Es ist gut und richtig, dass Deutschland einen solchen Weg nicht sucht, aber es ist offensichtlich nicht ausreichend, sich auf Verfassungspatriotismus und die europäischen Idee allein $\mathrm{zu}$ verlassen, denn „Die EU hat keine Erzählung“, so Hannes Stein in der „Welt“ vom 1. Juli 2016. Dieses Politikangebot als Leitidee für die Mitnahme der Bürger wird offensichtlich von vielen als nicht ausreichend empfunden.

\section{Umgang mit Minderheiten in Sachsen}

Die Konzentration auf Vorurteile und Negativberichte macht es manchmal unmöglich, auch das Positive zu sehen. Denn auch davon gäbe es in Sachsen viel zu berichten. So ist Sachsen neben Schleswig-Holstein (Dänen) und Brandenburg (Sorben) und gegebenenfalls Baden-Württemberg (in Heidelberg befinden sich der Sitz des Zentralrates Deutscher Sinti und Roma sowie das Dokumentations- und Kulturzentrum Deutscher Sinti und Roma) das Bundesland, in welchem eine traditionelle Minderheit lebt. Für Sachsen ergibt sich daraus die Aufgabe des Schutzes, der Bewahrung und Förderung von Minderheitenbelangen schon nach seiner Verfassung. Sachsen verfügt damit auch über besondere Erkenntnisse zum Umgang mit Menschen anderer Volksgruppen. Minderheitenschutz wird in Sachsen als ein Maßstab für die Integrationsfähigkeitund das Verantwortungsethos einer Gesellschaft gesehen. ${ }^{20}$ So erfolgt im „Gesetz über die Rechte der Sorben im Freistaat Sachsen“ in Paragraph 3 eine gemeindeteilgenaue Festschreibung des Sied-

\begin{tabular}{|l|c|c|c|c|c|c|c|}
\hline $\begin{array}{l}\text { Jahr/Stimmen- } \\
\text { anteil in Prozent }\end{array}$ & CDU & SPD & Linke & Grüne & FDP & NPD & AfD \\
\hline 1990 & 53,8 & 19,1 & 10,2 & 5,6 & 5,3 & 0,7 & - \\
\hline 1994 & 58,1 & 16,6 & 16,5 & 4,1 & 1,7 & - & - \\
\hline 1999 & 56,9 & 10,7 & 22,2 & 2,6 & 1,1 & 1,4 & - \\
\hline 2004 & 41,1 & 9,8 & 23,6 & 5,1 & 5,9 & 9,2 & - \\
\hline 2009 & 40,2 & 10,4 & 20,6 & 6,4 & 10,0 & 5,6 & - \\
\hline 2014 & 39,4 & 12,4 & 18,9 & 5,7 & 3,8 & 4,9 & 9,7 \\
\hline
\end{tabular}


lungsgebietes (im Gegensatz zu anderen Staaten unabhängig von prozentualen Bevölkerungsanteilen) für die Gültigkeit der Gesetzesnormen, was sich dann beispielsweise in Paragraph 9 ausformt in das (allerdings eingleisige) Recht des Gebrauchs der sorbischen Sprache im Siedlungsgebiet vor Gerichten und Behörden sowie öffentliche Einrichtungen unter staatlicher Aufsicht, weiter in die Umsetzung einer zweisprachige Beschilderung im Siedlungsgebiet, sorbisch sprechende Ansprechpartner, einen Rat für sorbische Angelegenheiten, die Institutionalisierung von Sorbenbeauftragten und die Abfassung eines Berichtes zur Lage des sorbischen Volkes mindestens einmal in jeder Legislaturperiode. Darauf aufbauend gibt es abgeleitete Verbindungen $\mathrm{zu}$ weiteren sächsischen Gesetzen wie dem „Schulgesetz für den Freistaat Sachsen“, das bestimmt, dass im „sorbischen Siedlungsgebiet [...] allen Kindern und Jugendlichen, deren Eltern es wünschen, die Möglichkeit zu geben“ ist, „die sorbische Sprache $\mathrm{zu}$ erlernen und in festzulegenden Fächern und Klassen- und Jahrgangsstufen in sorbischer Sprache unterrichtet zu werden.“ Auch sind an allen sächsischen Schulen Grundkenntnisse in Geschichte und Kultur der Sorben zu vermitteln. Zudem gibt es für die Sorben eine sehr langfristig angelegte Finanzierungsinstitution in Form einer Stiftung (mit ca. 16 Millionen Euro Jahresetat, wovon der Bund die Hälfte trägt, während ausgerichtet an der geschätzten Minderheitenverteilung sich Brandenburg mit einem Drittel und Sachsen mit zwei Dritteln die verbliebene andere Hälfte teilen).

Abschließend sei vermerkt, dass auch die Sächsische Staatsregierung verschiedene Projekte zur Willkommenskultur fördert und befördert. Zu denken wäre an die Ausstellung „Integration durch Leistung“, in der die porträtierten Persönlichkeiten ihre Erfahrungen mit Ankunft und Heimat wiedergeben - und zwar vom Vertriebenen über den Spätaussiedler, den vietnamesischen Gastarbeiter in der DDR bis hin zum heutigen Zuwanderer: „Ich habe mich engagiert, um meine neue Heimat mitzugestalten“ oder „Die freundliche Wesensart der Sachsen hat uns hier Freunde finden und heimisch werden lassen“ oder „Eigenes respektvolles Verhalten ist eine wichtige Voraussetzung für Integration“, lauten nur einige Meinungsbilder.

Egal, ob Deutscher, Sachse, Sorbe oder Migrant; alle suchen Heimat. Heimat lässt sich so auch mit dem Wunsch nach einem sicheren und sinnfälligen Leben in vertrauter Umge- bung assoziieren. Dazu muss der Einzelne durch Respekt gegenüber anderen beitragen. Umgekehrt bedingt Heimat aber auch, den Menschen eine Perspektive in seiner Region zu geben. Dies sollte von Politik und Verwaltung als Auftrag gesehen werden. Heimat ist zudem ohne Stabilität nicht vorstellbar. Von Menschen, die mit dem Verlassen ihres Landes eine Zeitgrenze, so Iris Radisch in der „Zeit“ vom 12. Mai 2016, überschreiten, weil sie aus einsichtigen Wünschen der Verspätung ihres Landes entfliehen wollen, muss man daher Integrationswillen erwarten dürfen und kein Verweigern, angefangen bei der Sprachbeherrschung. ${ }^{21}$ Ein Gegenteil von Dankbarkeit ist das falsche Zeichen; es ist vielmehr an der „Zeit, über eine andere Einwanderungspolitik nachzudenken“. ${ }^{22}$

\section{Schlussbetrachtung}

Für die hier wiedergegebenen Überlegungen hat der Autor mit mehreren Bekannten und Freunden aus den westlichen Bundesländern gesprochen, die seit langem in Sachsen leben. Immer wieder war der Verweis auf die jahrhundertealte kulturelle Tradition, die barocke Kulisse Dresdens und ein großes Traditionsverständnis zu hören. Einer meinte sogar, so offene Menschen wie hier in Sachsen habe er selten erlebt. Jeder hat seinen eigenen Umgang und eine solche Meinungssammlung ist keinesfalls repräsentativ. Aber das Sachsen anders sei, rechter, menschenfeindlicher das habe ich von keinem der hier Zugewanderten bisher gehört.

Mittlerweile wandelt sich ja auch das Bild in der Presse und damit in der Öffentlichkeit. Journalisten ${ }^{23}$ beginnen zu fragen, ob immer sachgemäß berichtet wurde. Nicht wenige Politiker stimmen der vertretenen Ansicht immer mehr zu, dass nicht alles richtig gelaufen war und der gesellschaftliche Zusammenhalt, der ja eine Stärke unseres Landes ist, in Gefahr gebracht werden könnte. So kann man nicht zuletzt den Maßnahmeplan für eine neue Sicherheitsstruktur vom Bundesinnenminister Dr. Thomas de Maizière, übrigens auch ein Sachse nach 1990, interpretieren. Die Politik hat das Problem, welches viele Menschen mit dem ungehinderten und nicht registrierten Zuzug von Flüchtlingen und Migranten haben, erkannt; eine rechtzeitige offene Diskussion darüber hätte wohl auch viel Unmut erspart. Vielleicht musste es aber auch so kommen, um Veränderungen $\mathrm{zu}$ erreichen. Deutlich ist auf jeden Fall geworden, dass die Bürger wach sind, alle Seiten.
20 Ausführlich bei Jens Baumann: Minderheitenförderung als Regionalförderung, in: Lětopis 1/2015.

21 Bassam Tibi, Die Welt, 4. Juli 2016.

22 Die Zeit, 29. Dezember 2016.

23 Hans Ulrich Gumbrecht, in: Die Zeit, 10. November 2016.

Es ist falsch zu glauben, dass der Mehrheitswille zu fairen Entscheidungen führt. Bloße Zahlen haben keine moralische Bedeutung. Eine Regierung ist nur legitim, wenn sie gleichen Respekt für alle zeigt - wenn also das Schicksal jeder Person gleich viel zählt.

Ronald Dworkin 
24 Welt N24, 17. Januar 2017: „Im Wortlaut des [Karlsruher NPD-]Urteils klingt das so: ,Es müssen konkrete Anhaltspunkte von Gewicht vorliegen, die es zumindest möglich erscheinen lassen, dass das Handeln der Partei erfolgreich sein kann (Potenzialität). Lässt das Handeln einer Partei dagegen noch nicht einmal auf die Möglichkeit eines Erreichens ihrer verfassungsfeindlichen Ziele schließen, bedarf es des präventiven Schutzes der Verfassung durch ein Parteiverbot nicht.' Ein Parteiverbot, so sagen es die Richter, sei nämlich kein ,Gesinnungs- oder Weltanschauungsverbot"“.

25 Lubomir Zaorálek, in: Die Welt, 15. Juni 2016.

26 Pascal Bruckner, in: Die Zeit, 14. April 2016.

27 Jürgen Habermas, in: Die Zeit, 7. Juli 2016.

28 Die Zeit, 3. März 2016.
Deutlich ist auch geworden, dass Stimmungen sich nicht unterdrücken lassen, dazu gibt es $\mathrm{zu}$ viele Foren, die bereit sind, ob richtig oder falsch, zu berichten. Auch Wahres kann falsch sein - indem man etwa im Fernsehen nur Frauen und Kinder als Flüchtlinge zeigt, verfälscht man genauso die Wahrheit, wie wenn man nur Straftaten durch Flüchtlinge aufzählt. Weiter ist deutlich geworden, dass durch Übertönen, Gegendemonstrieren und Totschweigen sich keine Probleme lösen lassen, hiervon wird auch niemand anders, höchstens innerlich verletzter, und das ist viel gefährlicher, als wenn er es wagt, seine Meinung zu sagen. Es ist ein Fakt derzeit, dass eine sowohl tatsächlich als auch im übertragenen Sinne laut agierende Minderheit das Gefühl des Einverständnisses der Mehrheit nach außen trägt. Es ist aber nicht die Aufgabe der Mehrheit, diese Minderheit zum buchstäblichen Schweigen zu bringen, denn Gedanken lassen sich eben nicht verbieten; man darf vielmehr erwarten, dass von außen Minderheit, Mehrheit und Meinungsfreiheit als jeweils eigenständig gesehen und Pauschalisierungen vermieden werden. Und nicht zuletzt hat sich wiederholt, was auch nach dem 3 . Oktober 1990 schon versucht worden ist: eine Partei, damals unter dem Namen PDS, durch Nichtbeachtung soweit zu diskreditieren, dass sie in den Augen der Wähler unmöglich gemacht wird, funktioniert nicht. Heute ist die PDS in manchen Ländern Koalitionspartner und unser Land ist dadurch nicht untergegangen. Widerspruch muss eine demokratische Gesellschaft aushalten ${ }^{24}$, und Widerspruch birgt auch manchmal ungeahnte Alternativen in sich, deren sich sogar im Zweifelsfall, weil es ja immer auch um Macht geht, die bereits Regierenden bedienen könnten.

Im Übrigen könnte man auch mit gleicher Berechtigung fragen, was denn in den westlichen Bundesländern anders ist. Der Blick kritischer Bürger Sachsens auf die deutsche Zuwanderungsdebatte mit dem verordneten „Wir schaffen das“ (aber will überhaupt jeder alles schaffen?) geht eher konform mit Positionen, die man auch als Regierungspolitik in Polen, Tschechien, der Slowakei oder in Ungarn findet, kurzum in den Visegrád-Staaten. ${ }^{25}$ Großbritannien wäre ebenso nicht $\mathrm{zu}$ vergessen, auch Frankreich teilt nicht die Ansichten der Bundesregierung. ${ }^{26}$ Wer ist denn dann anders - dies entscheidet sich je nach Blickrichtung. Und woher nimmt sich mancher das Recht der Befragungshoheit? Die deutsche Europapolitik wird nicht umsonst kritisiert: „Seit 2010 sehen wir, wie die deutsche Regierung die ihr ungewollt zugewachsene Führungsrolle in Europa weniger im gemeinsamen als im eigenen Interesse wahrnimmt. Deutschland ist ein widerwilliger, zugleich unsensibler und unfähiger Hegemon“. ${ }^{27}$ Ebenso wäre an Rüdiger Safranski oder Peter Sloterdijk zu denken, der anmerkte: „Merkel hat ganz einfach nicht das demokratische Mandat, ein Land so zu verändern". ${ }^{28}$

Bayern ist schwarz. Berlin ist rot. Die an der Küste halten Winterschlaf. Am Rhein feiert man immer noch Fastnacht. Die Brandenburger haben sich bei Senftenberg eine Indoorskihalle gebaut, um auch zum Abfahrtsski nicht mehr ihr schönes Land verlassen $\mathrm{zu}$ müssen. In Sachsen-Anhalt steht man früher auf, wozu auch immer. Auf dem Rennsteig gibt's auch bei 30 Grad plus Thüringer Klöße. Im Rheinland und in Baden-Württemberg ist ein Viertel immer noch ein Viertel, in Sachsen bekommt man für einen höheren Preis nur 0,2 Liter. Alle sind ein bisschen anders na und?! Deutschland ist bunt, wer bunt wollte, muss auch Schattierungen und Imre Kertész vertragen (Letzte Einkehr. Ein Tagebuchroman): „Zur Politik wäre noch das eine oder andere zusagen. [...] Es ginge darum, wie die Muslime Europa überschwemmen und in Besitz nehmen, direkt gesagt, zerstören werden. [...] Die Zivilisation erreicht einen überzüchteten Zustand, in dem sie nicht nur nicht mehr fähig, vielmehr auch nicht mehr willens ist, sich zu verteidigen; in dem sie, unverständlicherweise, ihre eigenen Feinde verherrlicht. Und dazu kommt, dass man das alles nicht öffentlich sagen darf. Wieso nicht?"

Manche Sachsen haben sich die Frage erlaubt, wieso auch nicht? Ob sie im richtigen Ton die richtige Antwort darauf haben, wer die richtige Antwort darauf hat, das müssen alle gemeinsam diskutieren. Und zwar diskutieren, ohne seine Meinung aufzuoktroyieren. Doch am Anfang steht die Frage, denn wie heißt es schon in den „Liedern des Kleinen Prinzen“ bei Kurt Demmler: „Frag und hast Du keine Fragen, so frag Dich warum.“ 